\title{
Assessment of a multiple biomarker panel for diagnosis of amyotrophic lateral sclerosis
}

\author{
Xueping Chen ${ }^{\dagger}$, Yongping Chen ${ }^{\dagger}$, Qianqian Wei, Ruwei Ou, Bei Cao, Bi Zhao and Hui-Fang Shang ${ }^{*}$
}

\begin{abstract}
Background: The aim of the study was to assess a panel of promising biomarkers for their ability to improve diagnosis of sporadic amyotrophic lateral sclerosis (ALS).

Methods: Forty patients with sporadic ALS and 40 controls with other neurological diseases were evaluated. Levels of phosphorylated neurofilament heavy chain (pNfH), S100- $\beta$, cystatin $C$, and chitotriosidase (CHIT) in cerebrospinal fluid were assayed using two-site solid-phase sandwich ELISA.

Results: Patients with sporadic ALS showed higher levels of pNfH and CHIT than controls, but lower levels of cystatin C. Multivariate logistic regression that adjusted for patient age and sex identified significant associations between sporadic ALS and levels of pNfH, CHIT and cystatin C. Levels of pNfH correlated positively with rate of progression and decline based on the Amyotrophic Lateral Sclerosis Functional Rating Scale - Revised. Based on receiver operating curve analysis, a pNfH cut-off of $437 \mathrm{ng} / \mathrm{L}$ discriminated patients from controls with a sensitivity of $97.3 \%$ and specificity of $83.8 \%$. A CHIT cut-off of $1593.779 \mathrm{ng} / \mathrm{L}$ discriminated patients from controls with a sensitivity of $83.8 \%$ and specificity of $81.1 \%$. Combining the two biomarkers gave a sensitivity of $83.8 \%$ and specificity of $91.9 \%$.
\end{abstract}

Conclusions: Levels of $\mathrm{pNfH}$ in cerebrospinal fluid may be a reliable biomarker for diagnosing ALS, and combining this biomarker with levels of CHIT may improve diagnostic accuracy.

Keywords: Amyotrophic lateral sclerosis, pNfH, S100- $\beta$, cystatin C, CHIT, Biomarker

\section{Background}

Amyotrophic lateral sclerosis is a fatal neurodegenerative disorder. It is diagnosed based on purely on clinical evaluation and differential diagnosis to exclude other possible conditions, highlighting the need to identify objective biomarkers that may aid diagnosis and perhaps help predict progression and prognosis.

Several candidate ALS biomarkers have been identified in cerebrospinal fluid (CSF), including neurofilament proteins, S100- $\beta$, cystatin C, and chitotriosidase (CHIT) $[1,2]$. The validity of these biomarkers remains controversial, since they were identified using targeted approaches rather than unbiased screens and they have

\footnotetext{
* Correspondence: hfshang2002@126.com

${ }^{\dagger}$ Equal contributors

Department of Neurology, West China Hospital, Sichuan University, Chengdu, Sichuan, China
}

(C) 2016 The Author(s). Open Access This article is distributed under the terms of the Creative Commons Attribution 4.0 International License (http://creativecommons.org/licenses/by/4.0/), which permits unrestricted use, distribution, and reproduction in any medium, provided you give appropriate credit to the original author(s) and the source, provide a link to the Creative Commons license, and indicate if changes were made. The Creative Commons Public Domain Dedication waiver (http://creativecommons.org/publicdomain/zero/1.0/) applies to the data made available in this article, unless otherwise stated. been assessed individually in separate studies, rather than in parallel. In addition, studies have not assessed the diagnostic potential of combinations of these candidate biomarkers.

Here we examined several candidate ALS biomarkers: phosphorylated neurofilament heavy chain ( $\mathrm{pNfH}$ ), S100- $\beta$, cystatin $C$, and CHIT. Our objective was to identify, in parallel comparisons, which biomarker(s) may be the most effective, as well as assess the diagnostic potential of biomarker combinations.

\section{Methods \\ Patients and samples \\ This study involved 40 patients clinically diagnosed with definite or probable sporadic ALS between May 2006 and November 2013 in the Department of Neurology at West China Hospital, Sichuan University (Chengdu, China). We}


also recruited 40 age- and sex- matched control patients diagnosed with non-ALS neurological diseases. These controls had lower motor neuron disease $(n=21)$, including spinal muscular atrophy $(n=8)$, multifocal motor neuropathy with conduction block $(n=6)$, spinal and bulbar muscular atrophy $(n=4)$, and chronic inflammatory demyelinating neuropathy $(n=3)$; or upper motor neuron disease $(n=19)$, including cervical myelopathy $(n=9)$, multiple sclerosis $(n=7)$, and hereditary spastic paraparesis $(n=3)$.

Motor and functional status of patients with sporadic ALS was quantified by neurologists using the Amyotrophic Lateral Sclerosis Functional Rating Scale - Revised (ALSFRS-R). 'Progression rate' was calculated from the equation: [(48 - ALSFRS-R score at baseline evaluation)/disease duration in months from symptom onset to evaluation]. Annual decline in ALSFRS-R was calculated from the equation: [(value at baseline examination - value at last examination)/years between the two examinations]. Monthly decline in ALSFRS-R was calculated by dividing the annual decline in ALSFRS-R by 12 .

CSF samples were obtained from all study participants by lumbar puncture. Samples were centrifuged at $3000 \mathrm{rpm}$ for $10 \mathrm{~min}$ at $4{ }^{\circ} \mathrm{C}$ to remove particulate matter. Protease inhibitor cocktail was added, and samples were aliquoted and stored at $-80{ }^{\circ} \mathrm{C}$ until analysis.

\section{ELISA}

Commercial two-site solid-phase sandwich ELISAs were used to assay levels of pNfH (RD191138300R, Biovendor), S100- $\beta$ (RD192090100R, Biovendor), cystatin C (RD191009100, Biovendor) and CHIT (cy-8074, MBL) in CSF samples. Kits were used according to the manufacturers' instructions. Before definitive measurements were taken, we assessed the stability of all four biomarkers in CSF by assaying their levels in samples that had been collected less than 30 min previously or more than $24 \mathrm{~h}$ previously and then stored at different temperatures. Biomarker levels remained stable after storage at $-80{ }^{\circ} \mathrm{C}$. Biomarkers did not appreciably aggregate under sample storage and thawing conditions, based on recovery experiments in which known amounts of biomarkers were added to the sample.

\section{Statistical analysis}

Data were reported as mean \pm standard deviation or median (range) and analyzed using SPSS 17.0 (IBM, Chicago, IL, USA). Since most data showed a skewed distribution, intergroup differences were assessed for significance using non-parametric statistical tests, i.e., the Mann-Whitney U and Kruskal-Wallis tests, followed by pair-wise post hoc tests corrected for multiple comparisons. The threshold of significance was defined as $p<0.05$.
Possible associations of sporadic ALS with levels of pNfH, S100- $\beta$, cystatin C, and CHIT in CSF were explored using multivariate logistic regression, with adjustment for age and sex as potential confounders. In this regression, biomarker levels were treated as categorical variables by stratification into quartiles based on the distributions observed in controls. The quartile with the lowest biomarker levels served as the reference group. Receiver operating curve (ROC) analysis was used to calculate an optimal cut-off level to discriminate patients with sporadic ALS from controls. Spearman rank correlation was used to explore possible correlations between demographic variables, disease duration, ALSFRS-R and progression rate on one hand and biomarker levels on the other. Patients were divided into various subgroups, and biomarker levels were compared between them using the Van Elteren test.

\section{Results}

Demographic and clinical characteristics of patients and controls are listed in Table 1. Most patients (33) had the spinal-onset form of the disease. Among all patients, mean rate of disease progression, in terms of ALSFRS-R score, was $0.91 /$ month. Twelve patients were defined as showing rapid progression because their monthly rate of progression was $>0.91$. Among all patients, mean rate of decline, again in terms of ALSFRS-R score, was 11.01/year, corresponding to a mean rate of decline of $0.92 /$ month. Ten patients were defined as showing rapid worsening because

Table 1 Demographic and clinical characteristics of patients with sporadic amyotrophic lateral sclerosis and controls with non-ALS neurological disorders

\begin{tabular}{lll}
\hline Characteristic & $\operatorname{ALS}(n=40)$ & $\begin{array}{l}\text { Controls } \\
(n=40)\end{array}$ \\
\hline Male, n (\%) & $26(55)$ & $24(60)$ \\
Age, yr & $52.08 \pm 11.45$ & $53.68 \pm 11.93$ \\
Age at onset, yr & $57.96 \pm 10.96$ & \\
Disease duration, mo & $17.61(2.77-50.43)$ & \\
Site of onset (spinal/bulbar), $n$ & $33 / 7$ & \\
ALSFRS-R score & $38.32(21-47)$ & \\
Progression rate & $0.91(0.08-5.06)$ & \\
$\begin{array}{l}\text { Rapidly progressive (progression } \\
\text { rate }>0.91 / \text { month)/Slowly progressive } \\
\text { (progression rate } \leq 0.91 / \text { month) }\end{array}$ & $12 / 28$ & \\
Annual decline of ALSFRS & & \\
Monthly decline of ALSFRS & $11.01(3.10-33.31)$ & \\
Rapidly worsening 0.92/month)/ & $10 / 16$ & \\
Slowly worsening (monthly & $26 / 11 / 3$ & \\
decline of ALSFRS-R $\leq 0.92 / m o n t h)$ & & \\
Survival/death/ lost to follow-up & $26 / 11 / 3$ & \\
\hline
\end{tabular}

Values shown are $\mathrm{n}, \mathrm{n}(\%)$, mean $\pm \mathrm{SD}$, or median (range) ALSFRS-R, Amyotrophic Lateral Sclerosis Functional Scale - Revised 
their monthly rate of decline was $>0.92$. Eleven patients died during follow-up, and three were lost to follow-up.

\section{Levels of pNfH, CHIT, cystatin C, and S100- $\beta$ in CSF}

Patients with sporadic ALS showed significantly higher levels of $\mathrm{pNfH}$ and CHIT in CSF than controls did $(P<0.0001 ;$ Fig. 1a-b). Conversely, patients with ALS showed a significantly lower level of cystatin $C$ $(P=0.0009$; Fig. 1c). Patients and controls did not differ significantly in levels of S100- $\beta$ (Fig. 1d).

\section{Associations of sporadic ALS with levels of pNfH, CHIT, cystatin C, or S100- $\beta$}

Multivariate logistic regression was used to explore possible associations of ALS status with levels of $\mathrm{pNfH}$, S100- $\beta$, cystatin C, or CHIT in CSF. The regression model adjusted for age and sex as possible confounders. Sporadic ALS was significantly associated with higher levels of pNfH, higher levels of CHIT, and lower levels of cystatin C. No significant correlation was identified between disease status and levels of S100- $\beta$.

Using ROC analysis, we calculated an optimal pNfH cut-off of $437 \mathrm{ng} / \mathrm{L}$ to discriminate patients with sporadic ALS from controls. This cut-off showed a sensitivity of $97.3 \%$ and specificity of $83.8 \%$; the area under the ROC curve (AUC) was 0.938 (95\% CI 0.884-0.991).
Using a CHIT cut-off of $1593.779 \mathrm{ng} / \mathrm{L}$ gave a sensitivity of $83.8 \%$, specificity of $81.1 \%$ and AUC of 0.854 (95\% CI 0.768-0.940; Fig. 2). Simultaneously applying both cut-offs gave a sensitivity of $83.8 \%$, specificity of $91.9 \%$ and AUC of 0.952 (95 \% CI 0.909-0.994.

\section{Associations of clinico-demographic characteristics with levels of pNfH, CHIT, and cystatin C}

Subgroups of patients with bulbar or spinal onset of sporadic ALS were similar in terms of $\mathrm{pNfH}$, CHIT, and cystatin $\mathrm{C}$ levels. Levels of $\mathrm{pNfH}$ were significantly higher in patients with rapidly progressing disease than in those with slowly progressing disease $(P=0.001)$, and they were higher in patients with rapidly worsening disease than in those with slowly worsening disease $(P=0.0143)$. Levels of CHIT and cystatin $C$ did not differ significantly between subgroups stratified by monthly rate of progression or decline. No significant associations were observed between ALSFRS-R score or disease duration on one hand and levels of the three biomarkers on the other (Table 2).

\section{Discussion}

In this study, we found that levels of $\mathrm{pNfH}$ in CSF were significantly higher in patients with sporadic ALS than

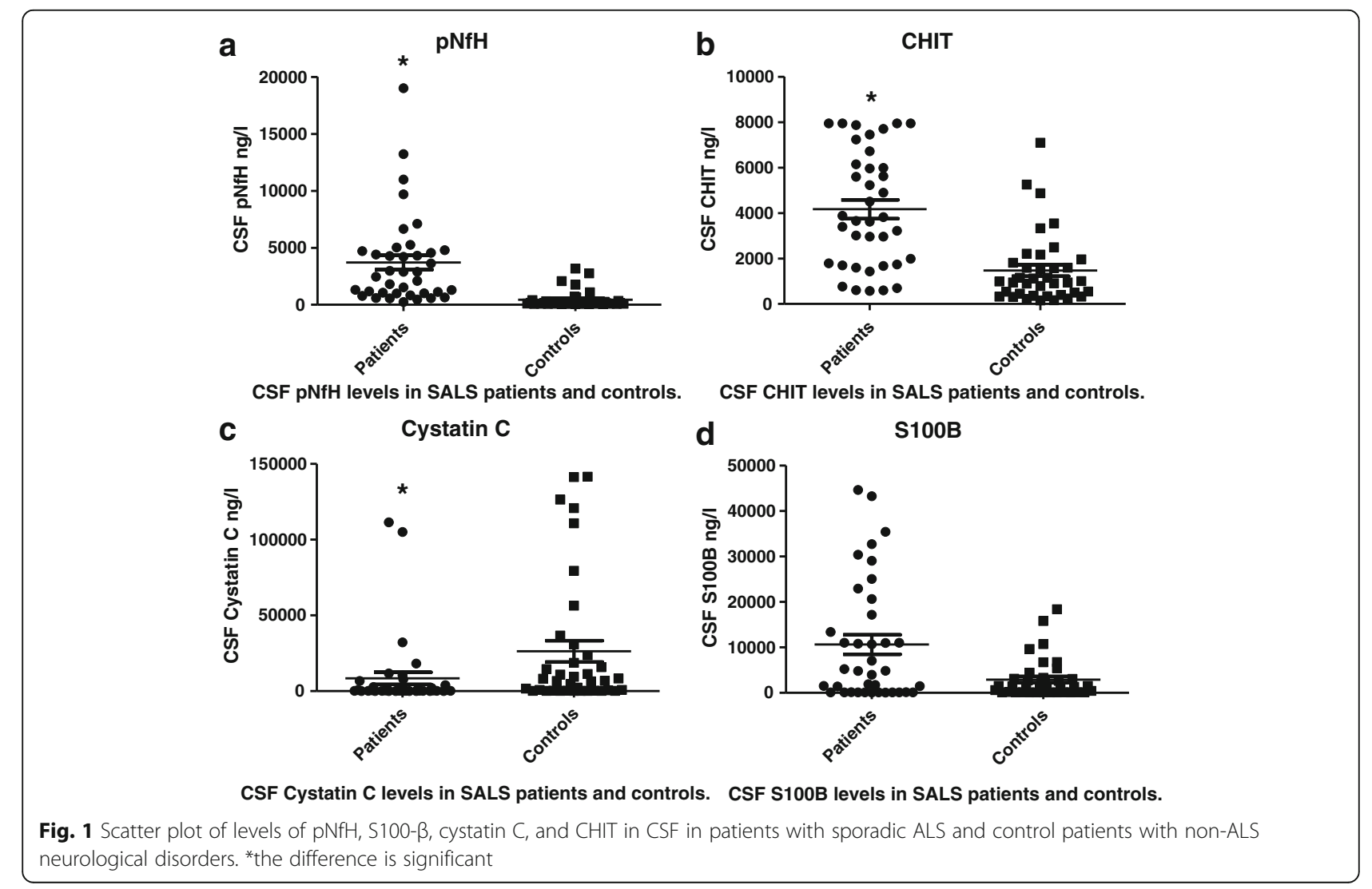




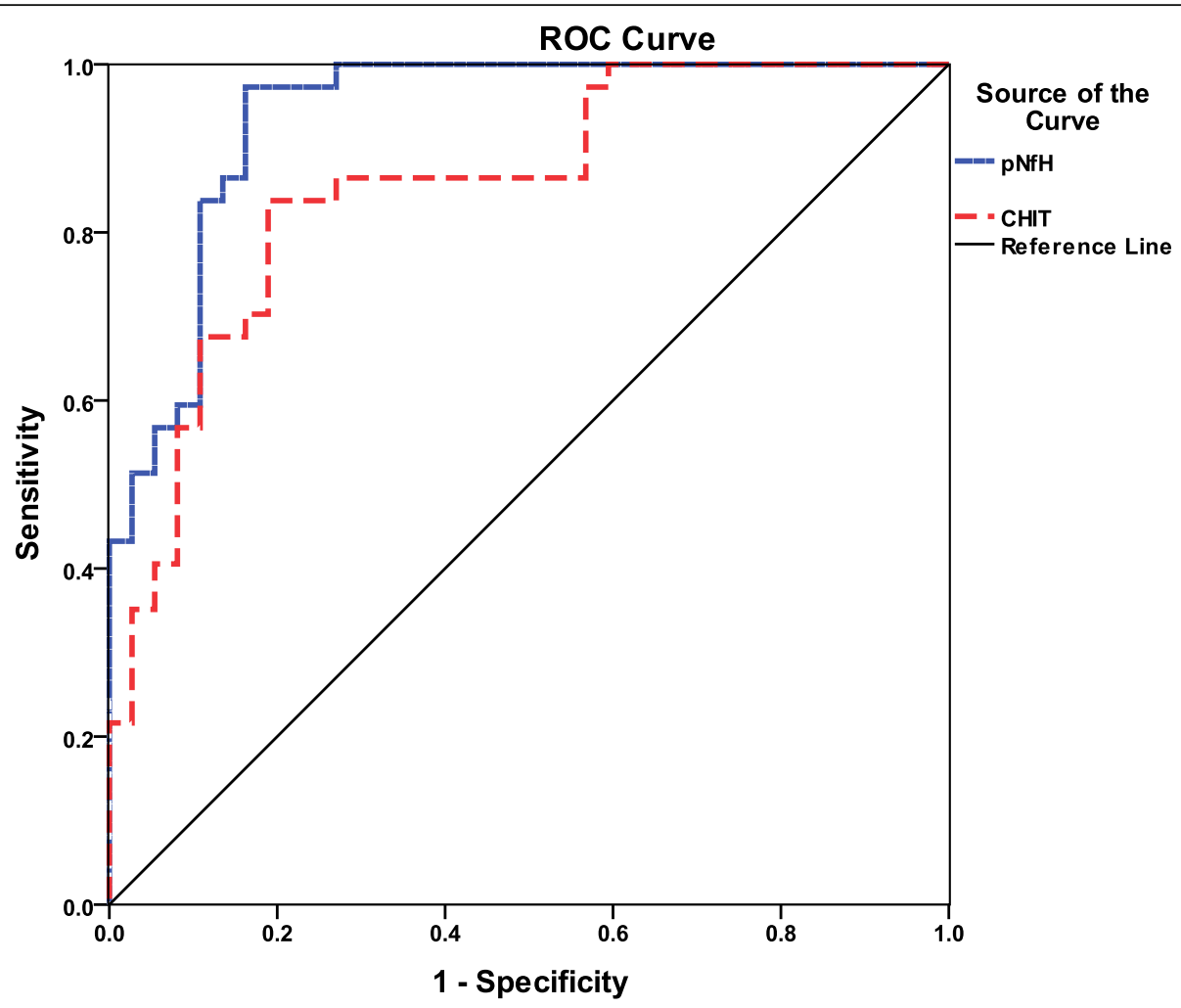

Fig. 2 Receiver operating curve (ROC) analysis

in controls with non-ALS neurological disorders. Similar results have been reported in other populations [3, 4]. In fact, Ganesalingam et al. reported 10-fold higher mean levels of pNfH in CSF in patients with ALS [5]. A large study of 455 patients reported significantly higher levels of $\mathrm{pNfH}$ in patients than in controls [6]. Multivariate regression of our data showed that sporadic ALS was associated with high levels of $\mathrm{pNfH}$ in CSF, even after adjusting for potential confounding variables. This suggests a possible role of $\mathrm{pNfH}$ in the pathogenesis of ALS, and it is consistent with studies showing that phosphorylation of $\mathrm{NfH}$ slows its axonal transport and interaction with other cytoskeletal proteins, affecting the course of ALS [7]. Higher levels of pNfH in the CSF of patients with ALS may reflect higher content of axonal proteins in motor neurons and greater extent of axonal injury, leading to the release of large amounts of $\mathrm{pNfH}$ into the extracellular space and ultimately into the CSF [8]. A recent study investigated CSF of 455 patients for $\mathrm{pNfH}$, and it also reported that $\mathrm{pNfH}$ levels were significant higher in ALS patients compared to the control groups [6].

Building on this observed association between sporadic ALS and levels of pNfH, we examined whether this

Table 2 Comparison of levels of pNfH, CHIT, and cystatin C in cerebrospinal fluid between subgroups of patients with sporadic ALS

\begin{tabular}{|c|c|c|c|c|c|c|}
\hline Subgroup & $\mathrm{pNfH}$ (ng/L) & $P$ value & CHIT (ng/L) & $P$ value & cystatin C (ng/L) & $P$ value \\
\hline \multicolumn{7}{|l|}{ Site of onset } \\
\hline Spinal & $4676(238-19002)$ & 0.43 & 4457 (572-7945) & 0.08 & $10330(62-111466)$ & 0.35 \\
\hline Bulbar & $3022(552-5265)$ & & 2886 (91-7945) & & 3065 (119-1113) & \\
\hline \multicolumn{7}{|l|}{ Progression rate } \\
\hline$>0.91 /$ month & 5969 (996-19002) & 0.001 & 3927 (572-7945) & 0.85 & $6566(62-105031)$ & 0.80 \\
\hline$\leq 0.91 /$ month & 1969 (238-5030) & & 3791 (91-7945) & & $12900(62-111466)$ & \\
\hline \multicolumn{7}{|l|}{ Worsening rate } \\
\hline$>0.92 /$ month & 6171 (1309-19002) & 0.0143 & $4668(598-7945)$ & 0.67 & $5188(78-32186)$ & 0.83 \\
\hline$\leq 0.92 /$ month & $2670(238-13223)$ & & $4243(572-7945)$ & & $15610(62-111466)$ & \\
\hline
\end{tabular}


biomarker shows diagnostic potential. Using a cut-off of $437 \mathrm{ng} / \mathrm{L}$ to discriminate between patients and controls gave sensitivity of $97.3 \%$ and specificity of $83.8 \%$. These results are consistent with other studies reporting relatively high sensitivity and specificity, although the optimal cut-off values vary substantially. Using a cut-off of $950 \mathrm{ng} / \mathrm{L}$, Brettschneider et al. reported sensitivity of $71 \%$ and specificity of $88 \%$ [3]. Using a cut-off of $502 \mathrm{ng} / \mathrm{L}$, Reijn et al. obtained sensitivity of $72 \%$ and specificity of $80 \%$ [4]. Steinacker et al. reported $83 \%$ sensitivity and $77 \%$ specificity with a cut-off of $560 \mathrm{ng} / \mathrm{L}$ [6]. Ganesalingam et al. reported sensitivity of $87.7 \%$ and specificity of $93.7 \%$ with a cut-off of $635 \mathrm{ng} / \mathrm{L}$ [9], while the same laboratory obtained respective values of $90 \%$ and $87 \%$ using a cut-off of $1200 \mathrm{ng} / \mathrm{L}$ in a larger patient sample [5]. This large variation in cut-off value may reflect the relatively small size of study populations, ethnic differences among the populations, and the intrinsic heterogeneity of sporadic ALS. The large variation may also reflect differences among the ELISA kits used. Given the strong evidence of diagnostic potential for $\mathrm{pNfH}$ levels in CSF, future work is urgently needed to develop and validate standard clinical biochemistry procedures for assaying this biomarker [10].

While pNfH levels are associated with presence of sporadic ALS, they do not appear to predict site of disease onset. In our study, levels were similar between subgroups of patients with bulbar or spinal onset. This is consistent with previous studies $[3,11]$.

We also investigated whether pNfH levels in CSF may show prognostic potential. We found that these levels did not correlate with clinical disability (ALSFRS-R score) at the time of diagnosis, consistent with a previous study [3]. However, these levels were significantly higher in our patients who suffered more rapid decline in ALSFRS-R score. In addition, the levels were significantly higher in patients with more rapidly progressive disease based on the progression rate [12, 13]. This echoes previous work associating elevated levels of $\mathrm{pNfH}$ with faster progression of ALS [3]. This suggests that $\mathrm{pNfH}$ levels may predict functional worsening in ALS. It may be that more aggressive disease is associated with increased, sustained cytoskeletal breakdown within motor neurons, which would lead to greater leakage of pNfH into CSF.

Our data also showed CHIT, an enzyme synthesized by microglia or infiltrating macrophages, to be present at significantly higher levels in CSF in patients with sporadic ALS than in controls. This is consistent with previous studies $[1,2]$. Higher CHIT levels may reflect a neuroinflammatory response triggered by microglia, since CHIT is considered a marker of microglial activation in stroke [14] and, like pro-inflammatory cytokines, an index of inflammation severity [15]. We further found that CHIT levels can distinguish patients from controls with sensitivity of $83.8 \%$ and specificity of $81.1 \%$, giving an acceptable AUC of 0.854 . To our knowledge, this is the first evidence that CHIT has diagnostic potential in sporadic ALS. At the same time, we found similar CHIT levels in subgroups of patients with rapidly or slowly progressing disease, in contrast to a previous study [2]. This may reflect clinical heterogeneity among patients with sporadic ALS.

We examined whether the combination of $\mathrm{pNfH}$ and CHIT levels may provide greater diagnostic accuracy than either biomarker alone. Using both gave a much higher specificity than pNfH alone (91.9\% vs. $83.8 \%$ ), but a lower sensitivity ( $83.8 \%$ vs. $97.3 \%)$. Our results suggest that combining $\mathrm{pNfH}$ and $\mathrm{CHIT}$ improves diagnostic specificity without sacrificing too much sensitivity.

We found that levels of cystatin C in CSF were significantly lower in patients with sporadic ALS than in controls. While several studies have reported a similar decrease in cystatin C levels in patients [16-19], analysis of samples from six centers in Europe failed to detect differences between patients and controls [20]. This discrepancy may reflect well-known preanalytic artifacts in assaying cystatin $C$ [21]. It may also reflect complex roles of cystatin $\mathrm{C}$ : this cysteine proteinase inhibitor has been implicated in both neuronal degeneration and neuronal repair. For example, Wilson et al. reported that cystatin C levels increase when ALS remains stable or progresses slowly, whereas levels decrease when disease progresses rapidly [22]. However, our data failed to indicate an association of cystatin $\mathrm{C}$ levels with disease progression, duration, or severity. Moreover, cystatin $\mathrm{C}$ levels could not distinguish patients from controls. Future studies should examine in greater detail the potentially complex role(s) of cystatin C in ALS.

We found no association between sporadic ALS and levels of S100- $\beta$ in CSF. This contrasts with several previous studies suggesting that this protein, which modulates the activity of effector proteins or cells, is present at higher levels in the CSF of patients with ALS [23-25]. Regardless of whether ALS involves changes in S100- $\beta$, assaying this protein is unlikely to be helpful for differential diagnosis of the disease, since levels of the protein have also been shown to change in other neurodegenerative disorders.

The results from our study should be interpreted with caution in light of at least three substantial limitations. One is our small sample, and another is the crosssectional design. Future work should monitor biomarker levels and disease onset and progression longitudinally. Third, our assays were not independently verified by an external laboratory.

Despite these limitations, the present study strengthens the evidence base that $\mathrm{pNfH}$ and CHIT are 
neuropathological hallmarks of ALS. Levels of $\mathrm{pNfH}$ and CHIT in CSF were significantly higher in patients than in controls, while levels of cystatin $\mathrm{C}$ were significantly lower in patients. In addition, pNfH levels in CSF correlated with disease severity and progression, and they discriminated patients from controls with high sensitivity and specificity. These findings point to $\mathrm{pNfH}$ as perhaps the single most promising candidate biomarker for ALS. At the same time, combining $\mathrm{pNfH}$ with CHIT may improve diagnostic accuracy. Given the heterogeneity of ALS, our results highlight the need for large longitudinal studies of patients with several disease phenotypes in order to verify and compare the ability of these biomarkers, alone and in combination, to differentiate ALS from related disorders.

\section{Conclusions}

Despite these limitations, the present study strengthens the evidence base that $\mathrm{pNfH}$ and CHIT are neuropathological hallmarks of ALS. Levels of pNfH and CHIT in CSF were significantly higher in patients than in controls, while levels of cystatin $\mathrm{C}$ were significantly lower in patients. In addition, pNfH levels in CSF correlated with disease severity and progression, and they discriminated patients from controls with high sensitivity and specificity. These findings point to $\mathrm{pNfH}$ as perhaps the single most promising candidate biomarker for ALS. At the same time, combining $\mathrm{pNfH}$ with CHIT may improve diagnostic accuracy. Given the heterogeneity of ALS, our results highlight the need for large longitudinal studies of patients with several disease phenotypes in order to verify and compare the ability of these biomarkers, alone and in combination, to differentiate ALS from related disorders.

\section{Abbreviations \\ ALS: Amyotrophic lateral sclerosis; ALSFRS-R: Amyotrophic Lateral Sclerosis Functional Rating Scale - Revised; AUC: Area under the ROC; CHIT: Chitotriosidase; CSF: Cerebrospinal fluid; pNfH: Phosphorylated neurofilament heavy chain; ROC: Receiver operating curve}

\section{Acknowledgments}

The authors thank the patients for their participation in the study.

\section{Funding}

The present study was supported by the National Natural Science Foundation of China (grant no. 81301093).

\section{Availability of data and materials}

The raw data summarized in this article are archived at West China Hospital. Although hospital policy prevents their public dissemination out of concern for patient privacy, individual requests for data access may be granted under appropriate circumstances. Interested parties should contact the authors.

\section{Authors' contributions}

XPC and YPC participated in study design and performed biochemical analyses. XPC drafted the manuscript. YPC performed statistical analysis. HFS conceived the study and assisted in study coordination and manuscript revision. QQW, RWO, BC, and BZ collected clinical data, including results of
CSF sample analysis, and participated in patient care and evaluation. All authors read and approved the final manuscript.

\section{Competing interests}

The authors declare that they have no competing interests.

\section{Consent for publication}

As part of their written informed consent to participate in this study, subjects also consented to the publication of their anonymized data for research purposes.

Ethics approval and consent to participate

The study protocol was approved by the Ethics Committee of West China Hospital, Sichuan University (approval no. 243). All participants provided written informed consent before being enrolled in the study.

Received: 13 March 2016 Accepted: 29 August 2016

Published online: 15 September 2016

\section{References}

1. Varghese AM, Sharma A, Mishra P, Vijayalakshmi K, Harsha HC, Sathyaprabha TN, Bharath SM, Nalini A, Alladi PA, Raju TR. Chitotriosidase - a putative biomarker for sporadic amyotrophic lateral sclerosis. Clin Proteomics. 2013;10(1):19.

2. Pagliardini $V$, Pagliardini $S$, Corrado L, Lucenti A, Panigati L, Bersano E Servo S, Cantello R, D'Alfonso S, Mazzini L. Chitotriosidase and lysosomal enzymes as potential biomarkers of disease progression in amyotrophic lateral sclerosis: a survey clinic-based study. J Neurol Sci. 2015:348(1-2):245-50.

3. Brettschneider J, Petzold A, Sussmuth SD, Ludolph AC, Tumani H. Axonal damage markers in cerebrospinal fluid are increased in ALS. Neurology. 2006:66(6):852-6.

4. Reijn TS, Abdo WF, Schelhaas HJ, Verbeek MM. CSF neurofilament protein analysis in the differential diagnosis of ALS. J Neurol. 2009;256(4):615-9.

5. Ganesalingam J, An J, Bowser R, Andersen PM, Shaw CE. pNfH is a promising biomarker for ALS. Amyotroph Lateral Scler Frontotemporal Degener. 2013;14(2):146-9.

6. Steinacker P, Feneberg E, Weishaupt J, Brettschneider J, Tumani H, Andersen PM, von Arnim CA, Bohm S, Kassubek J, Kubisch C, et al. Neurofilaments in the diagnosis of motoneuron diseases: a prospective study on 455 patients. J Neurol Neurosurg Psychiatry. 2016;87(1):12-20.

7. Shea TB, Jung C, Pant HC. Does neurofilament phosphorylation regulate axonal transport? Trends Neurosci. 2003;26(8):397-400.

8. Bowser R, Turner MR, Shefner J. Biomarkers in amyotrophic lateral sclerosis: opportunities and limitations. Nat Rev Neurol. 2011;7(11):631-8.

9. Ganesalingam J, An J, Shaw CE, Shaw G, Lacomis D, Bowser R. Combination of neurofilament heavy chain and complement C3 as CSF biomarkers for ALS. J Neurochem. 2011;117(3):528-37.

10. Otto M, Bowser R, Turner M, Berry J, Brettschneider J, Connor J, Costa J, Cudkowicz M, Glass J, Jahn O, et al. Roadmap and standard operating procedures for biobanking and discovery of neurochemical markers in ALS. Amyotroph Lateral Scler. 2012;13(1):1-10.

11. Boylan KB, Glass JD, Crook JE, Yang C, Thomas CS, Desaro P, Johnston A Overstreet K, Kelly C, Polak M, et al. Phosphorylated neurofilament heavy subunit (pNF-H) in peripheral blood and CSF as a potential prognostic biomarker in amyotrophic lateral sclerosis. J Neurol Neurosurg Psychiatry. 2013:84(4):467-72

12. Kimura F, Fujimura C, Ishida S, Nakajima H, Furutama D, Uehara H, Shinoda K, Sugino M, Hanafusa T. Progression rate of ALSFRS-R at time of diagnosis predicts survival time in ALS. Neurology. 2006;66(2):265-7.

13. Beghi E, Millul A, Logroscino G, Vitelli E, Micheli A. Outcome measures and prognostic indicators in patients with amyotrophic lateral sclerosis. Amyotroph Lateral Scler. 2008;9(3):163-7.

14. Sotgiu S, Barone R, Zanda B, Arru G, Fois ML, Arru A, Rosati G, Marchetti $B$, Musumeci S. Chitotriosidase in patients with acute ischemic stroke. Eur Neurol. 2005:54(3):149-53.

15. Di Rosa M, Malaguarnera G, De Gregorio C, D'Amico F, Mazzarino MC, Malaguarnera L. Modulation of chitotriosidase during macrophage differentiation. Cell Biochem Biophys. 2013;66(2):239-47. 
16. Mussap M, Plebani M. Biochemistry and clinical role of human cystatin C. Crit Rev Clin Lab Sci. 2004;41(5-6):467-550.

17. Nagai A, Ryu JK, Terashima M, Tanigawa Y, Wakabayashi K, McLarnon JG, Kobayashi S, Masuda J, Kim SU. Neuronal cell death induced by cystatin $C$ in vivo and in cultured human CNS neurons is inhibited with cathepsin B. Brain Res. 2005;1066(1-2):120-8.

18. Pasinetti $G M$, Ungar $L H$, Lange DJ, Yemul $S$, Deng $H$, Yuan $X$, Brown $R H$, Cudkowicz ME, Newhall K, Peskind E, et al. Identification of potential CSF biomarkers in ALS. Neurology. 2006;66(8):1218-22.

19. Ranganathan S, Williams E, Ganchev P, Gopalakrishnan V, Lacomis D, Urbinelli L, Newhall K, Cudkowicz ME, Brown Jr RH, Bowser R. Proteomic profiling of cerebrospinal fluid identifies biomarkers for amyotrophic lateral sclerosis. J Neurochem. 2005;95(5):1461-71.

20. Lehnert S, Costa J, de Carvalho M, Kirby J, Kuzma-Kozakiewicz M, Morelli C, Robberecht W, Shaw P, Silani V, Steinacker P, et al. Multicentre quality control evaluation of different biomarker candidates for amyotrophic lateral sclerosis. Amyotroph Lateral Scler Frontotemporal Degener. 2014;15(5-6):344-50.

21. Carrette O, Burkhard PR, Hughes S, Hochstrasser DF, Sanchez JC. Truncated cystatin C in cerebrospiral fluid: Technical [corrected] artefact or biological process? Proteomics. 2005;5(12):3060-5.

22. Wilson ME, Boumaza I, Lacomis D, Bowser R. Cystatin C: a candidate biomarker for amyotrophic lateral sclerosis. PLoS One. 2010;5(12):e15133.

23. Sussmuth SD, Sperfeld AD, Hinz A, Brettschneider J, Endruhn S, Ludolph AC Tumani H. CSF glial markers correlate with survival in amyotrophic lateral sclerosis. Neurology. 2010;74(12):982-7.

24. Boillee S, Yamanaka K, Lobsiger CS, Copeland NG, Jenkins NA, Kassiotis G, Kollias G, Cleveland DW. Onset and progression in inherited ALS determined by motor neurons and microglia. Science. 2006;312(5778):1389-92.

25. Yamanaka K, Chun SJ, Boillee S, Fujimori-Tonou N, Yamashita H, Gutmann DH, Takahashi R, Misawa H, Cleveland DW. Astrocytes as determinants of disease progression in inherited amyotrophic lateral sclerosis. Nat Neurosci. 2008:11(3):251-3.

\section{Submit your next manuscript to BioMed Central and we will help you at every step:}

- We accept pre-submission inquiries

- Our selector tool helps you to find the most relevant journal

- We provide round the clock customer support

- Convenient online submission

- Thorough peer review

- Inclusion in PubMed and all major indexing services

- Maximum visibility for your research

Submit your manuscript at www.biomedcentral.com/submit

) Biomed Central 